\title{
RINGS WITH ANNIHILATOR CHAIN CONDITIONS AND RIGHT DISTRIBUTIVE RINGS
}

\author{
MIGUEL FERRERO AND GÜNTER TÖRNER
}

(Communicated by Maurice Auslander)

\begin{abstract}
We prove that if a right distributive ring $R$, which has at least one completely prime ideal contained in the Jacobson radical, satisfies either a.c.c or d.c.c. on principal right annihilators, then the prime radical of $R$ is the right singular ideal of $R$ and is completely prime and nilpotent. These results generalize a theorem by Posner for right chain rings.
\end{abstract}

\section{INTRODUCTION}

The following question occurred in a paper by Posner [9]: Do there exist prime ideals in a right chain ring which are not completely prime? Several authors have approached this problem independently from various points of view (see [1]); however, the question remains open (see $[3,10])$, and it is natural to ask for additional conditions which imply that a prime ideal in a right chain ring is completely prime.

In the first part of [9, Theorem 2] it is claimed that if a right chain ring $R$ has either a.c.c. or d.c.c. on right annihilators, then the prime radical $P(R)$ of $R$ is the set of nilpotent elements. This fact implies that the prime radical of $R$ is completely prime. There is a gap in the proof and the chain conditions are needed for right ideals rather than right annihilator ideals, when $R$ is not prime. In fact, it is not proved that the annihilator chain conditions are inherited by $R / P(R)$; however, the result holds and the original motivation of this paper was to find a proof for it.

We say that a ring $R$ is a right distributive ring, or right $D$-ring for short, if its lattice of right ideals is distributive. It is well known that the class of commutative $D$-domains coincides with the class of Prüfer domains. The study of noncommutative right $D$-rings was mainly promoted by a paper of Stephenson [11]. The class of right chain rings (see [1] and the literature quoted therein) is an interesting class of examples. Brungs [2] proved that right $D$-domains are locally right chain rings. Recently two papers by Mazurek and Puczyłowski [8]

Received by the editors May 13, 1991 and, in revised form, February 21, 1992.

1991 Mathematics Subject Classification. Primary 16P60, 16U99; Secondary 16D30, 16N99.

This work was partially supported by a grant from Conselho Nacional de Desenvolvimento Científico e Tecnológico (CNPq, Brazil) and Gesellschaft für Mathematik und Datenverarbeitung (GMD, Germany). 
and Mazurek [7] showed that some features of right chain rings can be carried over to right $D$-rings.

The purpose of this note is to prove the following:

Theorem 8. Let $R$ be a right D-ring, which has at least one completely prime ideal contained in the Jacobson radical and satisfies either a.c.c. or d.c.c. on principal right annihilators. Then the prime radical of $R$ equals the right singular ideal of $R$ and is completely prime and nilpotent.

We say that the ring $R$ satisfies condition (C) if the following holds:

There exists a completely prime ideal $Q$ of $R$ contained in the Jacobson radical $J$ of $R$.

This condition first appeared in [11, Proposition 2.1(ii)]. Later it was used in [8], and in [7], where it was shown that the condition is of great interest. Let us point out that it is automatically satisfied for a right chain ring $R$; therefore, our result gives an extension of Posner's assertion.

Throughout this paper, every ring $R$ has a unit element. By $J=J(R)$ we denote the Jacobson radical, $P=P(R)$ the prime radical, and $A=A(R)$ the generalized nil radical of $R$. Further, we write $r(a)=\{x \mid a x=0\}$ the principal right annihilator of the element $a$ in $R$. The notations $\subset$ and $\supset$ will mean strict inclusions. Ideals are assumed to be two-sided unless otherwise stated.

\section{PROOF OF THE THEOREM}

Let $R$ be any ring. By $Z=Z_{r}(R)=\{x \in R \mid r(x)$ is an essential right ideal of $R$ \} we denote the right singular ideal of $R$ (see [5, pp. 30-36]).

An ideal $I$ of a ring $R$ is said to be right $T$-nilpotent if for every sequence $\left(x_{i}\right)_{i \in \mathbb{N}}$ of elements of $I$ there exists an $n$ such that $x_{n} x_{n-1} \cdots x_{2} x_{1}=0$. We begin with the following:

Lemma 1. Suppose that $R$ satisfies a.c.c. on principal right annihilators. Then $Z$ is right $T$-nilpotent, in particular, $Z \subseteq P(R)$.

Proof. Suppose $\left(x_{i}\right)_{i \in \mathbb{N}}$ is a sequence of elements in $Z$ such that $x_{n} \cdots x_{2} x_{1} \neq$ 0 for all $n \in \mathbb{N}$. Since $r\left(x_{1}\right) \subseteq r\left(x_{2} x_{1}\right) \subseteq \cdots$ is an ascending chain of principal right annihilators, there exists $m$ with $r\left(x_{m} b\right)=r(b), b=x_{m-1} \cdots x_{1}$. Now $x_{m}$ is in $Z$ and $b \neq 0$, so $r\left(x_{m}\right) \cap b R \neq 0$ and there exists $y \in R$ with $b y \neq 0$, $x_{m} b y=0$, which is a contradiction. The proof is complete since every ideal which is right $T$-nilpotent is contained in the prime radical (see [4, Proposition 2.3]).

We recall the following results [11, Proposition 2.1 (ii); 7, Lemma 3.1(ii), Corollary 3.3, and Theorem 3.4] for a right distributive ring $R$.

Lemma 2. Let $R$ be a right $D$-ring and $Q$ a completely prime ideal contained in $J$.

(i) For every right ideal $I$ of $R$ we have $I \subseteq Q$ or $Q \subseteq I$.

(ii) For any $a, b \in R$ we have: The elements $a, b$ are comparable, that is, $a R \subseteq b R$ or $b R \subset a R$ or otherwise $a Q=b Q$ holds.

(iii) The prime radical $P$ of $R$ is a prime ideal.

(iv) There is no two-sided ideal $I$ of $R$ with $P \subset I \subset A$. 
Note that from Lemma 2(i) condition (C) is satisfied in a right $D$-ring if and only if the generalized nil radical $A$ of $R$ is completely prime. This was already remarked in $[8$, p. 469]. Obviously this is automatically true provided $R$ is a right chain ring (see [1]).

Now we can prove the following:

Proposition 3. Let $R$ be a right D-ring which satisfies condition (C). Then $R$ is right nonsingular if and only if it is a domain.

Proof. Assume that $Z=0$ and let $Q$ be a completely prime ideal of $R$ contained in $J$. If $Q$ equals zero we are done. So we may assume $Q \neq 0$. Take any nonzero elements $a, b \in R$. By Lemma 2(ii) we have the alternatives $a R \subseteq b R, b R \subseteq a R$, or $a Q=b Q$. If $a Q=0$ holds, then $r(a) \supseteq Q$ and, by Lemma 2(i), $r(a)$ is an essential right ideal of $R$. Hence $a \in Z=0$. Therefore we have $a R \cap b R \neq 0$ and so the right Goldie dimension of $R$ is one. Thus $Z=0$ is a completely prime ideal of $R$ by [7, Proposition 1.2(i)]. Consequently, $R$ is a domain. The converse is obvious.

Mazurek pointed out to us the following lemma, which was proved by Tuganbaev in a more general setting [12, Lemma 8]. For the sake of completeness, we include an adaption of Tuganbaev's proof to our case.

Lemma 4. Let $R$ be a right $D$-ring. Then $R / Z$ has no nonzero nilpotent elements.

Proof. Assume that $a \notin Z$ and $a^{2} \in Z$. Then $H=r\left(a^{2}\right)$ is an essential right ideal of $R$ and $L=r(a)$ is not essential. So there exists a nonzero right ideal of $B$ of $R$ with $L \cap B=0$ and so $L \cap(H \cap B)=0$. By [11, Corollary 1(i) ${ }^{\prime}$ of Proposition 1.1] we have $\operatorname{Hom}_{R}(H \cap B, L)=0$, and since $a(H \cap B) \subseteq L$, we get $a(H \cap B)=0$. Therefore $H \cap B \subseteq r(a)=L$, hence $H \cap B=0$, a contradiction.

Now we prove some lemmas, which are necessary for the d.c.c. case.

Lemma 5. Let $R$ be a right D-ring which satisfies condition (C). If $I$ is an ideal of $R$ with $A \nsubseteq I$, then we have $I \subseteq P(R)$.

Proof. By Lemma 2(i), $I \subset A$. Hence, if $A=P$, we are done; therefore, we may assume $P \subset A$. Suppose there exists $a \in I$ with $a \notin P$, and take any element $b \in P$. Then one of the following three contradictions will follow: (i) $a \in b R \subseteq P$, or (ii) $a A=b A \subseteq P$, which contradicts the primeness of $P$, or (iii) $b \in a R \subseteq I$. The last possibility would imply $P \subseteq I \subset A$ and so $I=P$. Thus $I \subseteq P$.

Lemma 6. Let $R$ be a right $D$-ring and $Q$ a completely prime ideal contained in $J$. Then $Q^{2}=\{a b \mid a, b \in Q\}$.

Proof. By induction, it is enough to prove for $x=a_{1} b_{1}+a_{2} b_{2} \in Q^{2}$ with $a_{i}$, $b_{i} \in Q$ for $i=1,2$ that there exist $a, b \in Q$ with $x=a b$. By Lemma 2(ii) we have either $a_{1}=a_{2} y$ resp. $a_{2}=a_{1} y$, for some $y \in R$ or $a_{1} Q=a_{2} Q$. In the second case $a_{1} b_{1}=a_{2} b^{\prime}$ follows for some $b^{\prime} \in Q$. The rest is obvious.

Lemma 7. Let $R$ be a right $D$-ring and $Q$ a completely prime ideal of $R$ contained in $J$. Further assume $R$ satisfies d.c.c. on principal right annihilators. Then we have

(i) $Z \subseteq Q$;

(ii) If $Q=Q^{2} \neq 0$, then $Z \subset Q$. 
Proof. (i) If $Q=0$, then $R$ is a domain and $Z=0$. So we may assume $Q \neq 0$. Suppose $Q \subset Z$ and take any $a \in Z, a \notin Q$, and $0 \neq b \in Q$. By [7, Lemma 3.1(i)] we have $Q=a Q$. Hence there exists $c \in Q$ such that $b=a c$. So $r(c) \subseteq r(b)$ and $r(a) \cap c R \neq 0$. Thus we find $x \in R$ with $c x \neq 0$ and $a c x=0$, which implies $r(c) \subset r(b)$. Continuing in this way and starting with $c$ instead of $b$ we will reach a contradiction to the d.c.c. Therefore $Z \subseteq Q$, by Lemma 2 (i).

(ii) Assume $Q=Z$ and take any element $0 \neq a \in Q$. By assumption $a=b c$ for some $b, c \in Q$ (use Lemma 6). Hence $r(c) \subseteq r(a)$, and with the same arguments as in (i) we get $r(c) \subset r(a)$. This leads again to a contradiction as in (i). Therefore $Z \subset Q$.

Now we are able to prove Theorem 8 .

Proof. Case 1. Assume that $R$ satisfies a.c.c. on principal right annihilators. By the symmetric version of Theorem 2.2 and the final remark in [6], $R / P(R)$ is a right nonsingular right $D$-ring which satisfies $(C)$. Then $P(R)$ is completely prime by Proposition 3. Also, $Z=P(R)$ by Lemmas 1 and 4 . Finally by [7, Theorem 3.2], we have that $P$ is either nilpotent or $P=P^{2} \neq 0$. Assume $P=$ $P^{2} \neq 0$ and take any $0 \neq a \in P$. Then there exists $a_{1}, b_{1} \in P$ with $a=a_{1} b_{1}$. Repeating the argument, starting with $a_{1}$ instead of $a$, we have $a=a_{2} b_{2} b_{1}$ for some $a_{2}, b_{2} \in P$. By induction, we get a sequence $\left\{b_{1}, b_{2}, \ldots\right\}$ of elements of $P$ such that for every $m \geq 1$ there exists $a_{m} \in P$ with $a=a_{m} b_{m} \cdots b_{1}$. On the other hand $P=Z$ is right $T$-nilpotent, and so we get $a=0$, a contradiction.

Case 2. Assume that $R$ satisfies d.c.c. on principal right annihilators.

Since the generalized nil radical $A$ is completely prime, $Z \subseteq A$ by Lemma 7. So $Z \subseteq P$ if $A=P$. If $A \neq P$ holds, we have $A=A^{2} \neq 0$ by Lemma 2(iv) which implies $Z \subset A$ by Lemma 7 again. Thus, by Lemma $5, Z \subseteq P$ follows in any case. Therefore by Lemma $4 P=Z$ and $R / P$ is a prime ring which has no nonzero nilpotent elements. Consequently $P$ is completely prime. Finally, if $P$ is not nilpotent, as in Case 1 we have $P=P^{2} \neq 0$. So we get $Z \subset P$, a contradiction.

Corollary 9. Let $R$ be a right $D$-ring which satisfies condition (C) and a.c.c. on principal right annihilators. Then $P=N_{l}(R)$, where $N_{l}(R)$ is the set of left zero-divisors of $R$.

Proof. Obviously we have $P \subseteq N_{l}(R)$. Assume there exists $a \notin P$ with $r(a) \neq$ 0 . Hence $r(a) \subseteq r\left(a^{2}\right) \subseteq \cdots$ and $a^{n} \notin P$ for every integer $n$, since $P$ is completely prime. By assumption, there exists $m$ with $r\left(a^{m}\right)=r\left(a^{m+1}\right)$. Take any $0 \neq b \in r(a)$. Thus $a b=0$, and it follows that $b \in P \subset a^{m} R$; therefore, $b=a^{m} x$ for some $x \in R$, so $a^{m+1} x=0$, which leads to $b=a^{m} x=0$, a contradiction.

We were unable to answer the following

Question. Is $P=N_{l}(R)$ also under d.c.c. for principal right annihilators?

Obviously, it has an affirmative answer if $R$ is prime.

For the sake of completeness, we include a rather obvious example showing the relevance of assumption (C). 
Example 10. There exist right $D$-rings in which the prime radical is not a completely prime ideal, even under strong conditions of finiteness.

Let $K_{i}, i=1,2, \ldots, n$, be fields and set $R=K_{1} \oplus K_{2} \oplus \cdots \oplus K_{n}$. We denote by $e_{i}$ for $i=1, \ldots, n$ the canonical idempotent $(0, \ldots, 1, \ldots, 0)$. It is easy to check that every ideal of $R$ is of the type $R e$ with $e=e_{i_{1}}+\cdots+e_{i_{j}}$, for some idempotents $e_{i_{k}}$. So the lattice of ideals of $R$ is finite. By Theorem 1.6 in [11] $R$ is right distributive if and only if for every $a, b \in R$ there exist $x, y \in R$ with $b x \in a R, a y \in b R$, and $x+y=1$. Applying this result it can easily be deduced that the ring $R$ constructed above is right (and left) distributive. We have $J(R)=(0)$ and so $R$ does not satisfy the assumption $(C)$, since $(0)$ is neither completely prime nor prime. Thus the prime radical $P(R)=0$ is not prime. Moreover, we remark that any nonzero ideal of $R$ is idempotent and so there exist nonzero idempotent ideals, which are not prime provided $n \geq 3$. (We recall from [1] that in right chain rings idempotent ideals are always completely prime.)

\section{ACKNOWLEDGMENT}

We are grateful to C. Bessenrodt, A. Mazurek, and the referee for remarks that helped to improve the first version of the paper.

\section{REFERENCES}

1. C. Bessenrodt, H. H. Brungs, and G. Törner, Right chain rings, Part 1, Schriftenreihe des Fachbereichs Mathematik, Universität Duisburg, 1990.

2. H. H. Brungs, Rings with a distributive lattice of right ideals, J. Algebra 40 (1976), 392-400.

3. N. I. Dubrovin, An example of a nearly simple chain ring with nilpotent elements, Mat. USSR-Sb. 120 (1983), 441-447. (Russian)

4. B. J. Gardner, Some aspects of T-nilpotence, Pacific J. Math. 53 (1974), 117-130.

5. K. R. Goodearl, Ring theory, nonsingular rings and modules, Marcel Dekker, New York, 1976.

6. B. Johns, Chain conditions and nil ideals, J. Algebra 73 (1981), 287-294.

7. R. Mazurek, Distributive rings with Goldie dimension one, Comm. Algebra 19 (1991), 931944.

8. R. Mazurek and E. R. Puczyłowski, On nilpotent elements of distributive rings, Comm. Algebra 18 (1990), 463-471.

9. E. Posner, Left valuation rings and simple radical rings, Trans. Amer. Math. Soc. 107 (1963), 458-465.

10. M. Schröder, Über N. I. Dubrovin's Ansatz zur Konstruktion von nicht vollprimen Primidealen in Kettenringen, Results in Math. 17 (1990), 296-306.

11. W. Stephenson, Modules whose lattice of submodules is distributive, Proc. London Math. Soc. (3) 28 (1974), 291-310.

12. A. A. Tuganbaev, Rings in which the structure of right ideals is right distributive, Soviet Math. (Iz. VUZ) 30 (1986), 58-65.

Instituto de Matemática, Universidade Federal do Rio Grande do Sul, 90049 Porto ALEGRE, BRAZIL

E-mail address, M. Ferrero: ferrero@if1.ufrgs.br

Fachbereich Mathematik, Universität Duisburg, Postfach 101503, 4100 Duisburg, GERMANY

E-mail address, G. Törner: toerner@math.uni-duisburg.de 